Research paper

\title{
Orbital flips due to solar radiation pressure for space debris in near-circular orbits
}

\author{
S.O. Belkin ${ }^{\mathrm{a}}$, E.D. Kuznetsov ${ }^{\mathrm{b}, *}$ \\ ${ }^{a}$ Moscow Institute of Physics and Technology, 9 Institutskiy per., Dolgoprudny, Moscow Region, 141701, Russian Federation \\ ${ }^{\mathrm{b}}$ Ural Federal University, 51 Lenina Avenue, Yekaterinburg, 620000, Russian Federation
}

\section{A R T I C L E I N F O}

\section{Keywords:}

Space debris

Orbital flip

Global navigation satellite systems

Disposal orbits

Secondary apsidal-nodal secular resonance

\begin{abstract}
A B S T R A C T
Orbital plane flips, a transition from prograde to retrograde motion or vice versa, is a phenomenon due to solar radiation pressure that is investigated. We consider initial near-circular orbits with different inclinations, including the vicinity of orbits of the GNSS satellites, GEO, geosynchronous orbits, and super-GEO region. Dynamical evolution of orbits is studied from a numerical simulation. Initial conditions for the objects are chosen in the GNSS orbit regions (GLONASS, GPS, BeiDou, Galileo) as well as 450-1100 km above to nominal semi-major axes of the navigation orbits, and in the vicinity of GEO, geosynchronous orbits, and super-GEO region. Initial data correspond to nearly circular orbits with the eccentricity 0.001 . The initial inclination is varied from $55^{\circ}$ to $64.8^{\circ}$. Initial values of longitude of ascending node are varied from $0^{\circ}$ to $350^{\circ}$. High area-to-mass ratios are considered, at which orbital plane flips occur. Dynamical evolution covers periods of 24 and 240 years. The maximum inclination of the orbit is achieved when the longitude of the pericenter is sun-synchronous. Flips are possible only for objects with the area-to-mass ratio equal or more than $16 \mathrm{~m}^{2} / \mathrm{kg}$ (the radiation pressure coefficient is 1.44). The flips are caused precisely by solar radiation pressure. The Lidov-Kozai effect is suppressed by solar radiation pressure perturbations, affecting high area-to-mass ratio objects due to a secondary apsidal-nodal secular resonance.
\end{abstract}

\section{Introduction}

The transition from prograde motion, when the inclination of the orbit $i$ is less than $90^{\circ}$, to retrograde motion with orbital inclination more than $90^{\circ}$ or vice versa is called flip. This phenomenon can occur due to various factors, one of which, for example, is the Lidov-Kozai effect [1]. In [2], while studying the dynamic properties of orbits that can be used to store satellites that have completed their intended purpose, it was shown that flips of the orbit plane due to light pressure are observed in the vicinity of the orbits of the satellites of global navigation systems. However, just the same effect, i.e. the orbit flip due to high solar radiation pressure, had already been shown for GPS satellites in $[3,4]$. The effect of the solar radiation pressure for orbits around the Earth was considered mainly in the perspective of bodies characterized by a very high area-to-mass ratio. We can mention works focused on the orbital evolution of Geostationary Earth Orbits (GEO) (e.g. [5-9]) and Medium Earth Orbits (MEO) (e.g. [10-15]).

In this work, a more detailed study of flips in the vicinity of the navigation satellite motion region is carried out. This implies a description of the methods used to obtain results on modeling the motion of artificial Earth satellites under the influence of various kinds of disturbances. Emphasis is placed on the effect of light pressure since we consider objects with a large area-to-mass ratio (about $15-80 \mathrm{~m}^{2} / \mathrm{kg}$ ). Also, in the paper, we present the results of modeling under different initial conditions and varying such parameters of the studied object and its orbit as area-to-mass ratio, the longitude of the ascending node, the major axis, and the initial inclination. The "Results" section also provides a phenomenological description of the results obtained in order to explain the connection of flips with light pressure.

The study of this phenomenon is valuable nowadays due to intensive development of near-Earth space. For efficient in the long term work of the satellites, it is necessary to take into account all the perturbations that may affect them. Also it is necessary to ensure the safety of currently operating apparatuses from collisions with space debris. To provide it, satellites that have completed their intended purposes can in particular be redirected to orbits subject to smaller variations in order to reduce the probability of their collision with operating satellites.

\footnotetext{
* Corresponding author.

E-mail addresses: astroboy96@mail.ru (S.O. Belkin), eduard.kuznetsov@urfu.ru (E.D. Kuznetsov).
} 
Table 1

Argument $\psi_{j}=n_{1} \Omega+n_{2} g+n_{3} \lambda_{S}$ of the periodic component in terms of $n_{1}, n_{2}, n_{3}$.

\begin{tabular}{llrr}
\hline$j$ & $n_{1}$ & $n_{2}$ & $n_{3}$ \\
\hline 1 & 1 & 1 & -1 \\
2 & 1 & -1 & -1 \\
3 & 0 & 1 & -1 \\
4 & 0 & 1 & 1 \\
5 & 1 & 1 & 1 \\
6 & 1 & -1 & 1 \\
\hline
\end{tabular}

\section{Methods}

\subsection{Singly averaged model}

Before embarking on a numerical simulation of the orbital evolution of an object in the vicinity of the Earth, we consider the averaged equations of motion. The averaged model will highlight the most significant combinations of angular elements that determine the main features of the Earth's satellite's dynamical evolution under the influence of the solar radiation pressure. Let us assume that the radiation coming from the Sun is directed normally to the surface of the object (e.g., a satellite or space debris fragment), that is, the so-called cannonball model. Moreover, let us consider the orbit of the spacecraft entirely in the sunlight, and the effect of Earth's albedo as negligible. In the following, ( $a, e, i, \Omega, g$ ) are the Keplerian orbital elements of the object measured with respect to the Earth equatorial plane (namely, the semi-major axis, the eccentricity, the inclination, the longitude of the ascending node, the argument of the pericenter). Neglecting the light aberration, the solar radiation pressure is a conservative force. The corresponding disturbing potential, averaged over the orbital motion of the object, can be written as (e.g. $[16,17])$

$\mathcal{R}_{S R P}=\frac{3}{2}$ Pkrae $\sum_{j=1}^{6} \mathcal{T}_{j} \cos \psi_{j}$,

where $P$ the solar radiation pressure, $k$ the reflectivity coefficient and $\gamma$ the area-to-mass ratio,

$\psi_{j}=n_{1} \Omega+n_{2} g+n_{3} \lambda_{S}$,

with $n_{1}=0,1, n_{2}= \pm 1, n 3= \pm 1$, according to $\mathrm{j}$, following Table 1 , where $\lambda_{S}$ is the longitude of the Sun measured on the ecliptic plane, and

$\mathcal{T}_{1}=\cos ^{2}\left(\frac{\epsilon}{2}\right) \cos ^{2}\left(\frac{i}{2}\right)$,

$\mathcal{T}_{2}=\cos ^{2}\left(\frac{\epsilon}{2}\right) \sin ^{2}\left(\frac{i}{2}\right)$,

$\mathcal{T}_{3}=\frac{1}{2} \sin (\epsilon) \sin (i)$,

$\mathcal{T}_{4}=-\frac{1}{2} \sin (\epsilon) \sin (i)$,

$\mathcal{T}_{5}=\sin ^{2}\left(\frac{\epsilon}{2}\right) \cos ^{2}\left(\frac{i}{2}\right)$,

$\mathcal{T}_{6}=\sin ^{2}\left(\frac{\epsilon}{2}\right) \sin ^{2}\left(\frac{i}{2}\right)$,

where $\epsilon$ is the obliquity of the ecliptic.

We consider a simple dynamical model. Let us assume that an object moves under the effect of the Earth's gravitational monopole, the Earth's oblateness, and the solar radiation pressure. For the solar radiation pressure, it is assumed in the cannonball model that the orbit of the spacecraft is entirely in sunlight and that the effect of the Earth's albedo is negligible (e.g., $[15,16])$. The singly averaged the Lagrange equations of motion of the Earth' satellite can be written for positional elements of the orbit $(a, e, i)$ as

$\frac{d a}{d t}=0$
Table 2

GNSS orbits and potential disposal orbits.

\begin{tabular}{lllllll}
\hline \multirow{2}{*}{ GNSS } & GNSS orbit & & & \multicolumn{2}{l}{ Disposal orbit } & \multirow{2}{*}{$i_{\circ}$, deg } \\
\cline { 2 - 3 } & Resonance & $a_{\text {GNSS }}, \mathrm{km}$ & & Resonance & $a_{d}, \mathrm{~km}$ & \\
\hline GLONASS & $8: 17$ & 25508 & & $14: 29$ & 25947 & 64.8 \\
GPS & $1: 2$ & 26559 & & $15: 29$ & 27168 & 55 \\
BeiDou & $7: 13$ & 27907 & & $4: 7$ & 29034 & 55 \\
Galileo & $10: 17$ & 29600 & & $3: 5$ & 29994 & 56 \\
\hline
\end{tabular}

$\frac{d e}{d t}=-\frac{3}{2} P k \gamma \frac{\sqrt{1-e^{2}}}{n a} \sum_{j=1}^{6} \mathcal{T}_{j} \frac{\partial \cos \psi_{j}}{\partial g}$

$\frac{d i}{d t}=-\frac{3}{2} P k \gamma \frac{e}{n a \sqrt{1-e^{2}} \sin i} \sum_{j=1}^{6} \mathcal{T}_{j}\left(\frac{\partial \cos \psi_{j}}{\partial \Omega}-\cos i \frac{\partial \cos \psi_{j}}{\partial g}\right)$,

where $n$ is the mean motion of the object.

In case of a resonance when the following condition is satisfied

$\dot{\psi}_{j}=0$,

only one term will dominate in the Eqs. (4). Resonances (5) arising in this case (see Table 1) can be considered as secondary apsidal-nodal and apsidal secular resonances (see e.g., [12,18-20]).

\subsection{Numerical simulation}

The dynamical evolution of space debris is studied using a numerical simulation. Initial conditions for the space objects in medium Earth orbits are chosen for the GNSS regions (GLONASS, GPS, BeiDou, Galileo) and orbits $450-1100 \mathrm{~km}$ above with respect to nominal semimajor axes of the navigation orbits $a_{G N S S}$ (Table 2). Here $a_{d}$ is the semi-major axis of the disposal orbit, which is chosen as a resonant with the Earth rotation. Such resonances do not play any role in the features that we are going to describe hereafter, as the variations of the Keplerian variables, namely the semi-major axis and the eccentricity and inclination couple, are not affected by the same perturbations. We use the resonant semi-major axis $a_{d}$ for disposal orbits to maintain continuity with the article [2].

The initial conditions take values corresponding to the studied orbits. Initial values of semi-major axes are varied from $25500 \mathrm{~km}$ (near the orbits of the GLONASS) to $30000 \mathrm{~km}$ (above orbits of Galileo) and $42200 \mathrm{~km}$ (near GEO). The initial value of the inclination depends on the navigation system and varies from $55^{\circ}$ and $56^{\circ}$ to $64.8^{\circ}$. The initial value of the eccentricity corresponds to a near-circular orbit with $e=0.001$. Initial values of the longitude of the ascending node $\Omega$ are varied from $0^{\circ}$ to $360^{\circ}$. The initial value of the argument of pericenter is $g=270^{\circ}$. The pericenter is directed toward the Sun when $\Omega=270^{\circ}$. The direction of the Sun is normal with respect to the orbital plane when $\Omega=0^{\circ}$ and $180^{\circ}$. The area-to-mass ratio $\gamma$ is chosen on the range of values from 8 to $80 \mathrm{~m}^{2} / \mathrm{kg}$. These large values of the area-to-mass ratio correspond to a large number of space debris. The dynamical evolution covers periods of 24 and 240 years. 24 years is enough to observe any local inhomogeneities, and a period of 240 years is enough to determine global dependencies in the resulting graphs. Initial epoch $T_{0}$ is $00^{\mathrm{h}} 00^{\mathrm{m}} 00^{\mathrm{s}} \mathrm{UTC}^{1} 21.03 .1958$.

The dynamic evolution of space debris in the vicinity of the area of motion of satellites of global navigation systems is studied based on numerical simulation. The orbital evolution of space objects is modeled in "Numerical Model of the Motion of an Artificial Satellites" [12,21] developed at the Tomsk State University. The model of perturbing forces takes into account the major perturbing factors:

1 We use UTC time scale since it corresponds to the format of the initial data of the numerical model. 


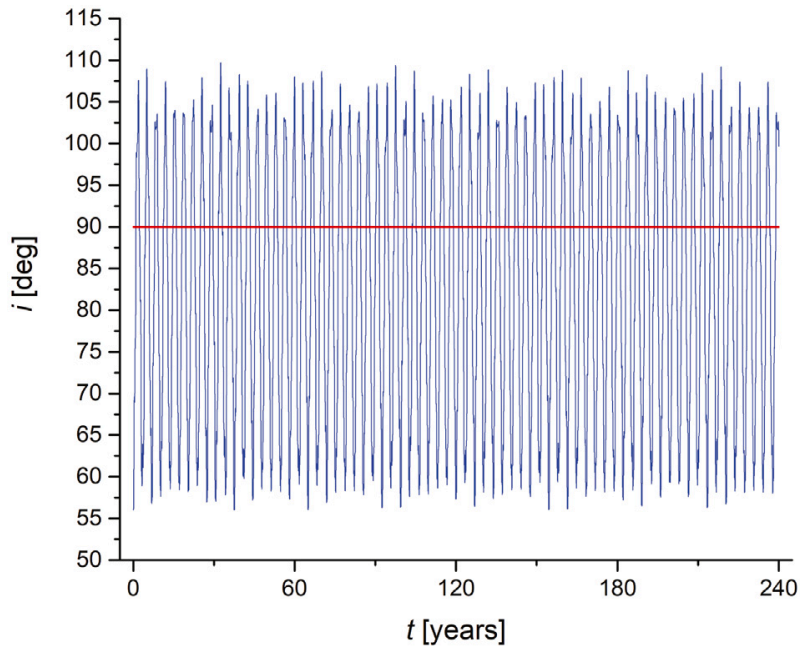

Fig. 1. Evolution of the orbital inclination $i$ for a satellite above Galileo orbit near the $3: 5$ resonance region for the area-to-mass ratio $\gamma=60 \mathrm{~m}^{2} / \mathrm{kg}\left(a_{\circ}=29994 \mathrm{~km}\right.$, $i_{\text {。 }}=56^{\circ}, k \gamma=86.4 \mathrm{~m}^{2} / \mathrm{kg}$ ).

- the gravitational field of the Earth (EGM96 model [22], harmonics up to the 29th order and degree, inclusive, for the correct description of the movement of objects in eccentric orbits and before re-entering),

- the gravitation of the Moon and the Sun,

- the tides of the Earth,

- the direct solar radiation pressure (the radiation pressure coefficient $k=1.44$ ) including the eclipses due to the Earth's shadow (the Earth's shadow duration can rich up to $0.07-0.08$ revolution for GNSS orbits and 0.05 for GEO),

- the Poynting-Robertson effect,

- and the atmospheric drag, using the Russian Federation National Standard GOST R 25645.166-2004 "Earth Upper Atmosphere" (for induced high eccentricity orbits with a perigee altitude of below $1500 \mathrm{~km}$, when eccentricity is more than 0.7 for GNSS orbits and 0.81 for GEO).

The equations of motion are integrated by Everhart's method of the 19th order [23]. The coordinates of the Moon and the Sun during the numerical integration of the equations of motion are extracted from the JPL Planetary and Lunar Ephemerides DE405/LE405 [24].

\section{Results}

\subsection{Orbital flips}

As a result of numerical modeling, we obtained a large number of model trajectories describing the motion of artificial Earth satellites, from which information was subsequently extracted to determine the presence of a flip for certain initial parameters of the orbit or the object under study. As we can see in the graphs given below, there is a relationship between the orbital flips and such parameters like longitude of the ascending node, area-to-mass ratio and the value of the semi-major axis. For orbits above Galileo near $3: 5$ resonance area with the initial parameters of the orbit and the object such as initial value of semi-major axis is $29994 \mathrm{~km}$, longitude of the ascending node is $180^{\circ}$, area-to-mass ratio is $\gamma=60 \mathrm{~m}^{2} / \mathrm{kg}\left(\mathrm{k \gamma}=86.4 \mathrm{~m}^{2} / \mathrm{kg}\right)$ and initial orbital inclination $56^{\circ}$, the dependence of the orbital inclination on time will be like in Fig. 1 .

We also can see in Fig. 1 that chosen object will spend almost $1 / 3$ of its time in retrograde motion. The period of orbital flips is approximately 3 years. The maximum inclination is about $108^{\circ}$.
For a better view of flips and evolution of the orbital inclination, we shorten the time of modeling from 240 to 24 years (see Fig. 2). Fig. 2 shows us how an orbital inclination evolves in time with different values of the ascending node. The total time in retrograde motion is approximately half of the whole time of modeling with a period of approximately 10 years. This means that during a significant part of the entire time of movement, the studied object moves in the retrograde regime. The amplitude of the inclination is about $110^{\circ}$. In this case, fragments of space debris are close to moving in orbits "opposite" to the orbits of the GLONASS satellites depending as well on the values of the longitude of the ascending node. That is, the movement will occur almost in the same orbital plane, but towards the movement of the active satellites of the system, which, together with a long time the object under study moves in the opposite direction, will pose a threat to the regular functioning of the system.

It is also noticeable the presence of some additional disturbances at $150^{\circ}$ (Fig. 2(a)) and $210^{\circ}$ (Fig. 2(c)) and its absence at $180^{\circ}$ (Fig. 2(b)). Extra perturbations in the inclination oscillations are manifested at values close to critical inclination, which is associated with zero rate of the apogee drift due to the second zonal harmonic of the Earth gravitational field. It is also could be seen in Fig. 2.

Figs. 3(a) and 3(c) for satellites of the GLONASS's orbits show that the eccentricity does not exceed the value of 0.55 (for used initial conditions) with the initial values of the ascending node of $150^{\circ}$ and $210^{\circ}$ with defined initial values of the semi-major axis and inclination, and high area-to-mass ratio $\gamma=35 \mathrm{~m}^{2} / \mathrm{kg}\left(k \gamma=50.4 \mathrm{~m}^{2} / \mathrm{kg}\right)$. That fact proves that we are dealing not with the Lidov-Kozai effect, which requires the presence of a large eccentricity during the flip. The same situation is in Fig. 3(b) where the initial value of the ascending node is $180^{\circ}$. The eccentricity does not exceed 0.4 , because of the condition, which was presented in [25] and will be described below. Also note that the proximity of the inclination to the critical value provides a slow motion of the pericenter, which, with proper selection of the initial conditions, allows to limit the eccentricity's amplitude.

The effect of the eccentricity's amplitude limitation is risen by equality between the initial longitude of pericenter $\pi=\Omega+g$ and the longitude of the Sun $\lambda_{S}$. In [25] the existence of a stationary point $\left(e_{\circ}\right.$, $\pi_{\circ}$ ) was demonstrated in the phase plane "eccentricity $e$ and longitude of pericenter $\pi$ ", corresponding to the following initial conditions

$e_{0}=\frac{3}{2} k \gamma P \frac{\cos ^{2} \epsilon / 2}{a n n_{S}} \approx 0.01 k \gamma, \quad \pi_{0}=\lambda_{S}$.

Here $P=4.56 \cdot 10^{-6} \mathrm{~N} \mathrm{~m}^{-2}$ is the radiation pressure, $\epsilon$ is the obliquity of the ecliptic to the equator, $n$ and $n_{S}$ are mean motions of a satellite and the Sun, and $\lambda_{S}$ is the ecliptic longitude of the Sun. The initial conditions corresponding to $\pi_{0}=\lambda_{S}$ in (6) are realized at $\Omega=180^{\circ}$ (Fig. 3(b)). The fulfillment of the condition ensures the eccentricity's amplitude limitation depending on the initial value of the eccentricity specified by $e_{0} \approx 0.01 k \gamma$ in (6). Here in cases Figs. 3(a) and 3(c), we can also see modulation and oscillation due to any perturbations present, which are absent at the longitude of the ascending node $180^{\circ}$.

To explain the features of the dynamical evolution of objects with a high area-to-mass ratio $\gamma$, we consider the behavior of the arguments $\psi_{j}(2)$ that appear on the right-hand sides of the averaged equations of motion (4). Fig. 4 shows the evolution of the arguments $\psi_{1}, \ldots, \psi_{6}$. The argument $\psi_{1}$ librates near $0^{\circ}$ with magnitude $90^{\circ}$ (Fig. 4(a)). Other arguments circulate from $0^{\circ}$ to $360^{\circ}$ with different rates (Fig. 4(b),Fig. 4(f)). Note that for all orbits from Table 2, the evolution of the $\psi_{1}, \ldots, \psi_{6}$ arguments occurs in a similar Fig. 4.

The resonance condition (5) for $j=1$ corresponds to prograde orbits, when the longitude of the pericenter is sun-synchronous [15]. We have classified the resonance as a secondary apsidal-nodal secular resonance. The primary apsidal-nodal secular resonance has the critical argument $\psi_{1}^{1} \equiv \pi=\Omega+g$. For initial Value of the longitude of the ascending node $\Omega_{0}=180^{\circ}$ mean value of the resonance argument $\psi_{1} \approx 0$ leads to condition $\pi=\Omega+g \approx \lambda_{S}$ which corresponds to 


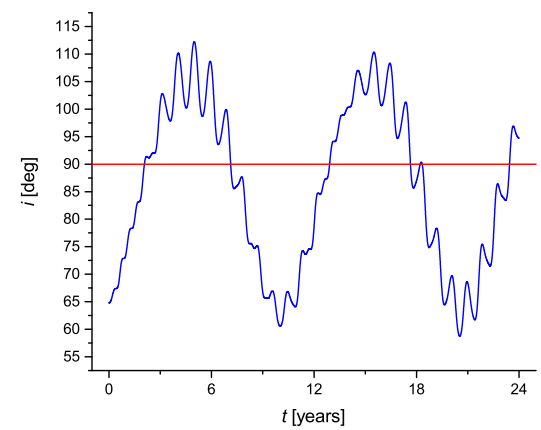

(a)

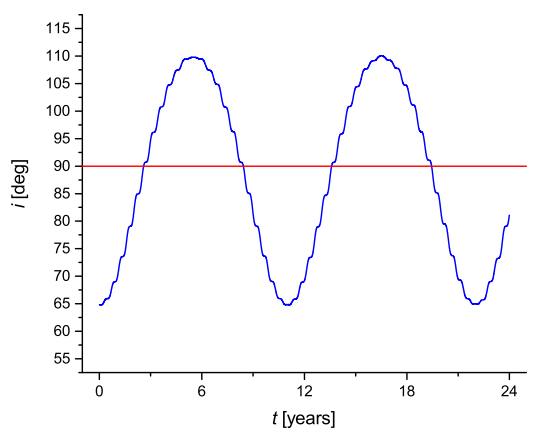

(b)

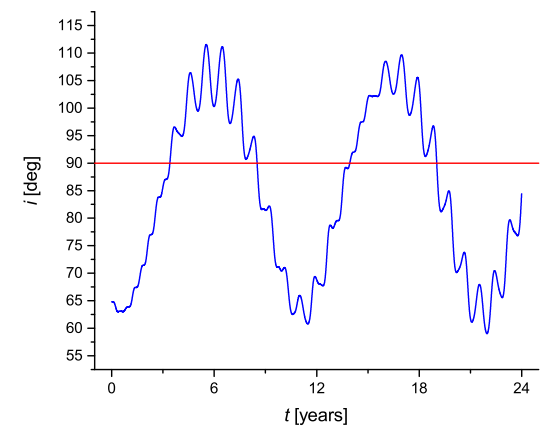

(c)

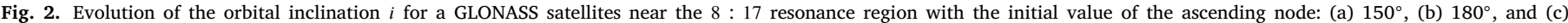
$210^{\circ}\left(a=25508 \mathrm{~km}, i=64.8^{\circ}, \gamma=35 \mathrm{~m}^{2} / \mathrm{kg}\left(k \gamma=50.4 \mathrm{~m}^{2} / \mathrm{kg}\right)\right.$ ).

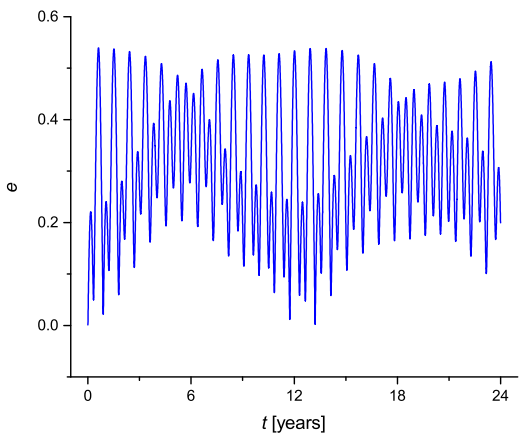

(a)

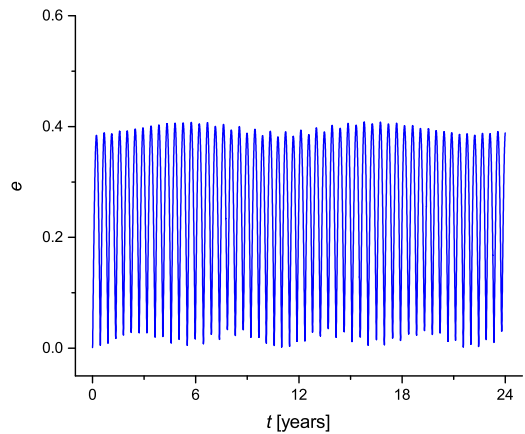

(b)

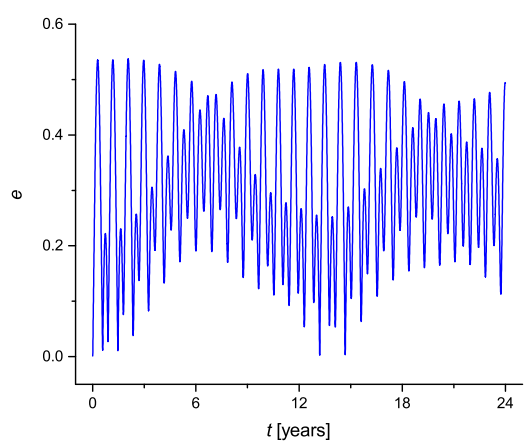

(c)

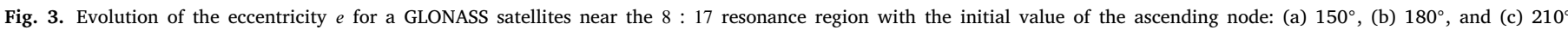
$\left(a=25508 \mathrm{~km}, i=64.8^{\circ}, \gamma=35 \mathrm{~m}^{2} / \mathrm{kg}\left(k \gamma=50.4 \mathrm{~m}^{2} / \mathrm{kg}\right)\right.$ ). 


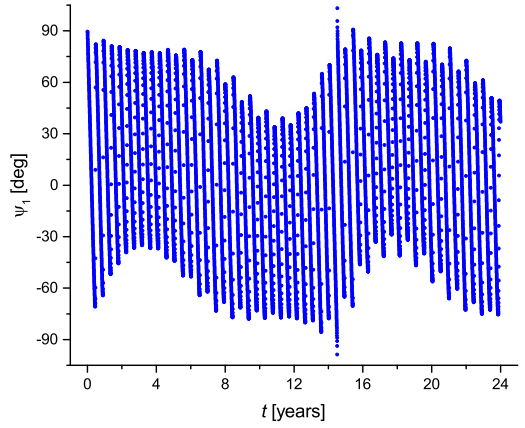

(a)

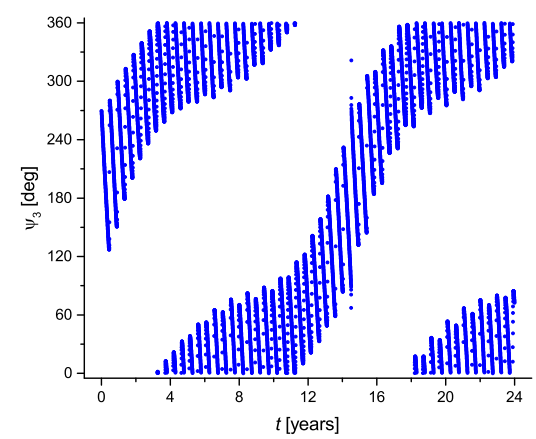

(c)

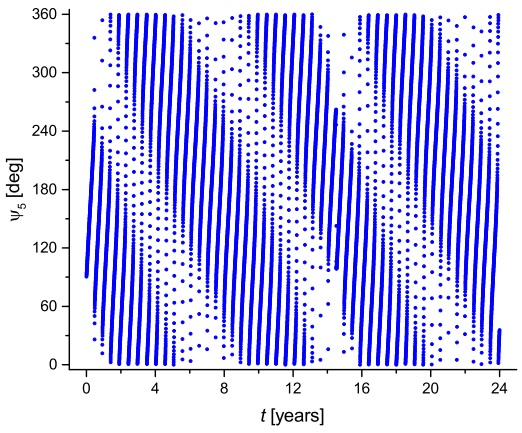

(e)

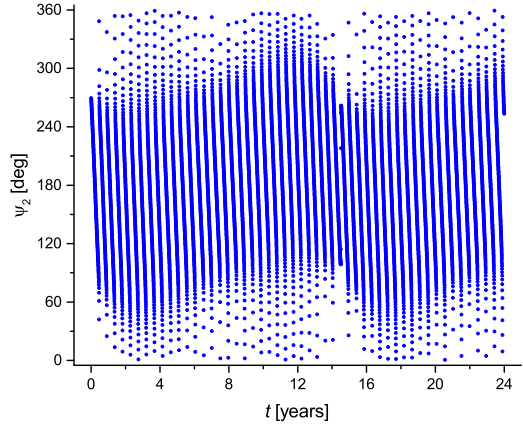

(b)

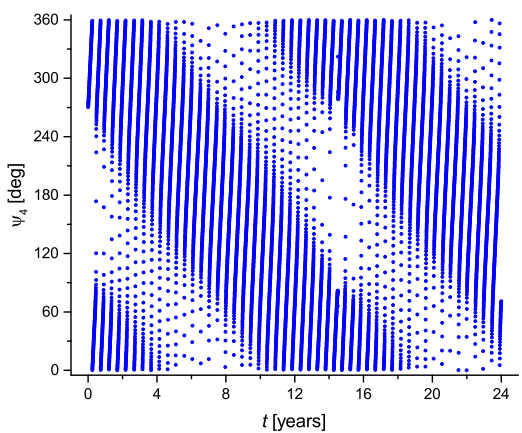

(d)

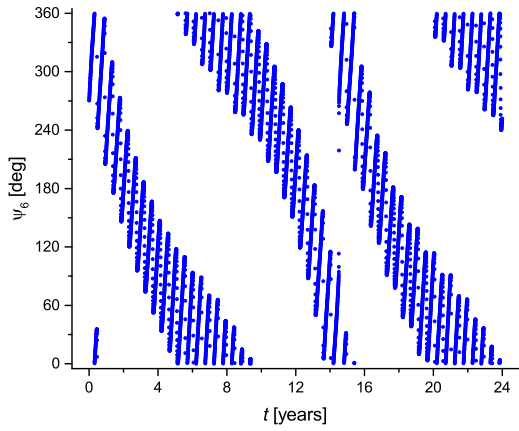

(f)

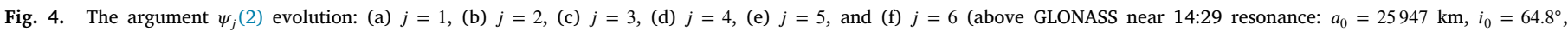
$\Omega_{0}=180^{\circ}, \gamma=30 \mathrm{~m}^{2} / \mathrm{kg}\left(k \gamma=43.2 \mathrm{~m}^{2} / \mathrm{kg}\right)$.

second expression in (6). The eccentricity as a function of the resonance argument $\psi_{1}$ evolves in libration mode (Fig. 5).

When the longitude of the pericenter is not sun-synchronous: $\pi \nsim \lambda_{S}$ (e.g., $\Omega \approx 180^{\circ}$ ), the orbital evolution is non-resonant (Figs. 6). The argument $\psi_{1}$ circulates, and the range of the eccentricity oscillation rises.

As shown in Fig. 7, the evolution of the inclination and the eccentricity occurs concordantly. Could the variations of the inclination and eccentricity be a demonstration of the Lidov-Kozai effect?

\subsection{The Lidov-Kozai effect}

Classical works [26] and [27] describe the effect in the quadrupole approximation of the double-averaged Restricted Three-Body Problem.

The Lidov-Kozai Hamiltonian is

$H=-\frac{G m_{1} a^{2}}{8 a_{1}^{3}}\left[2+3 e^{2}-3\left(1-e^{2}+5 e^{2} \sin ^{2} g\right) \sin ^{2} i\right]$.
Here $m_{1}$ is the mass of the perturbing body, $a_{1}$ is the semi-major axis of the orbit of the perturbing body, $a, e, i$ and $g$ are the semi-major axis, eccentricity, inclination, and argument of pericenter of the third body's orbit, respectively.

The Hamiltonian (7) has three integrals.

$c_{0} \equiv a=$ const,

$c_{1} \equiv\left(1-e^{2}\right) \cos ^{2} i=$ const,

$c_{2} \equiv e^{2}\left(\frac{2}{5}-\sin ^{2} i \sin ^{2} g\right)=$ const.

The types of motion of the third body depend on values of $c_{1}$ and $c_{2}(8)$. At $c_{2}<0$ the orbits have the argument of pericenter $g$ librating. At $c_{2}>0$ the orbits have the argument of pericenter circulating. The librating orbits exist only if $0<c_{1}<3 / 5$. The libration of the argument of pericenter $g$ takes place around either $\pi / 2$ or $3 \pi / 2$. The libration of the argument of pericenter entails the coupled variations in inclination $i$ and eccentricity $e$. 


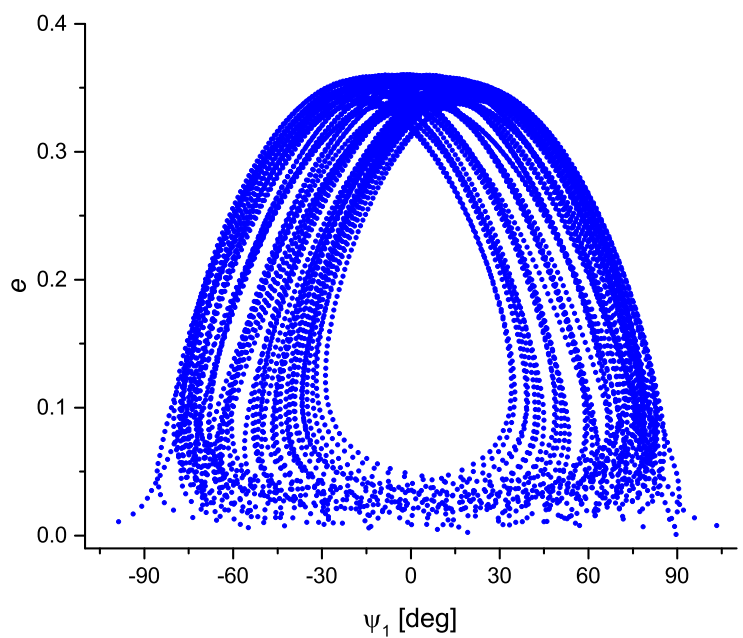

Fig. 5. Eccentricity as a function of resonance argument $\psi_{1}$ (above GLONASS near 14:29 resonance: $a_{0}=25947 \mathrm{~km}, i_{0}=64.8^{\circ}, \Omega_{0}=180^{\circ}, \gamma=30 \mathrm{~m}{ }^{2} / \mathrm{kg}\left(k \gamma=43.2 \mathrm{~m}^{2} / \mathrm{kg}\right.$ )).

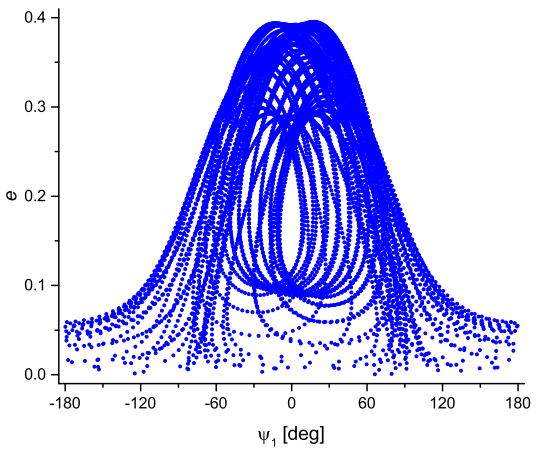

(a)

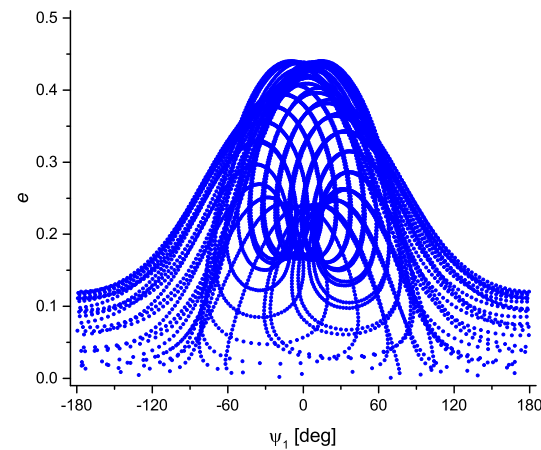

(b)

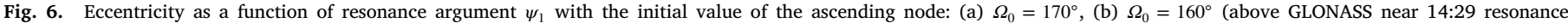
$\left.a_{0}=25947 \mathrm{~km}, i_{0}=64.8^{\circ}, \gamma=30 \mathrm{~m}^{2} / \mathrm{kg}\left(k \gamma=43.2 \mathrm{~m}^{2} / \mathrm{kg}\right)\right)$.

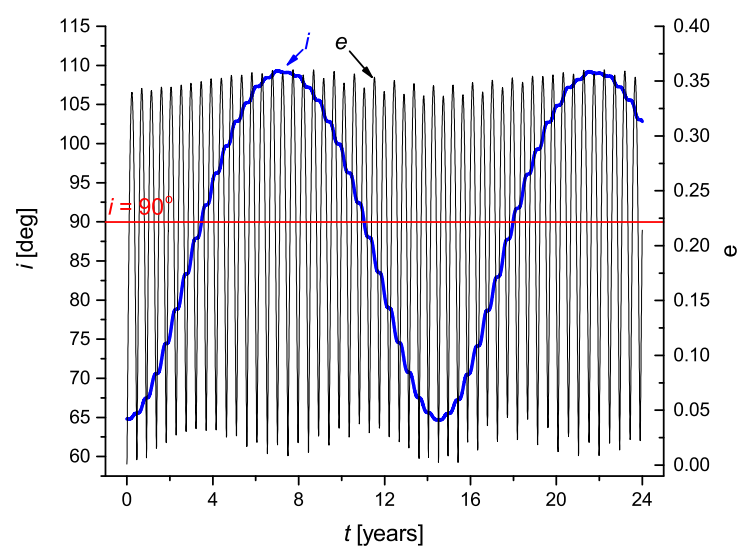

Fig. 7. Inclination $i$ (bold blue line) and eccentricity $e$ (black line) as functions of time $t$ (above GLONASS near 14:29 resonance: $a_{0}=25947 \mathrm{~km}, i_{0}=64.8^{\circ}, \Omega_{0}=180^{\circ}$, $\gamma=30 \mathrm{~m}^{2} / \mathrm{kg}\left(k \gamma=43.2 \mathrm{~m}^{2} / \mathrm{kg}\right)$ ). (For interpretation of the references to color in this figure legend, the reader is referred to the web version of this article.)

Integral $c_{1}$ in (8) is essentially the $z$ component of the angular momentum squared. Obviously, $0 \leq c_{1} \leq 1$. Besides, the constancy of $c_{1}$ means that (1) the secular variations of $e$ and $i$ are coupled in anti-phase

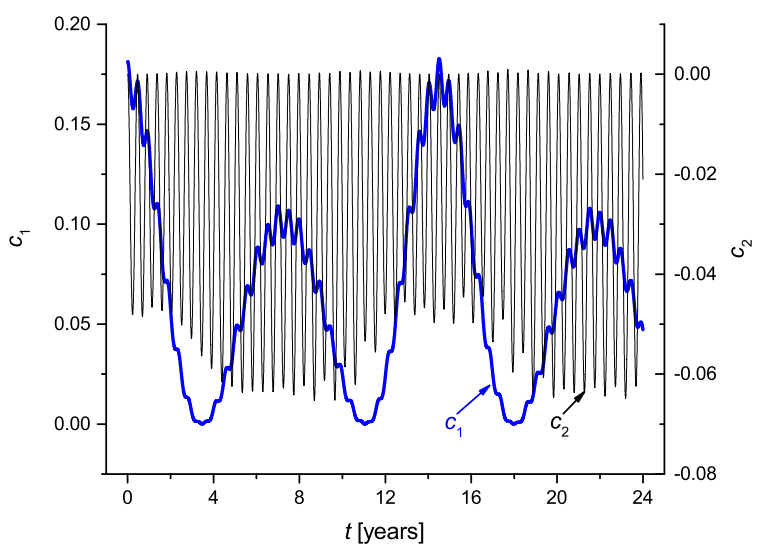

Fig. 8. Integrals $c_{1}$ (bold blue line) and $c_{2}$ (black line) as functions of time $t$ (above GLONASS near 14:29 resonance: $a_{0}=25947 \mathrm{~km}, i_{0}=64.8^{\circ}, \Omega_{0}=180^{\circ}, \gamma=30 \mathrm{~m}^{2} / \mathrm{kg}$ $\left(k \gamma=43.2 \mathrm{~m}^{2} / \mathrm{kg}\right.$ )). (For interpretation of the references to color in this figure legend, the reader is referred to the web version of this article.)

if $0 \leq i \leq \pi / 2$, and (2) the variations of $e$ and $i$ are coupled in phase if $\pi / 2 \leq i \leq \pi[1]$.

Fig. 8 shows that the integrals $c_{1}$ and $c_{2}(8)$ are preserved with low accuracy and vary widely. The conditions $0<c_{1}<3 / 5$ and $c_{2}<0$ are almost always realized and the argument of pericenter $g$ librates. 


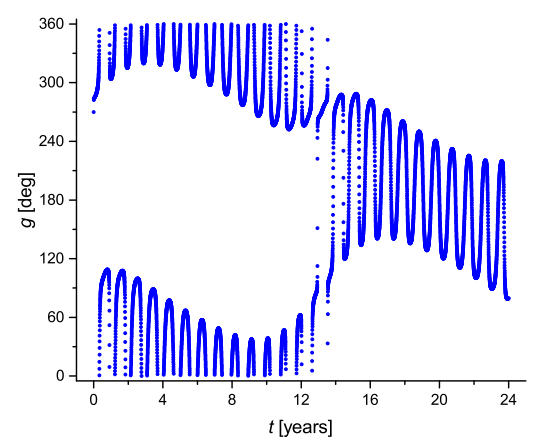

(a)

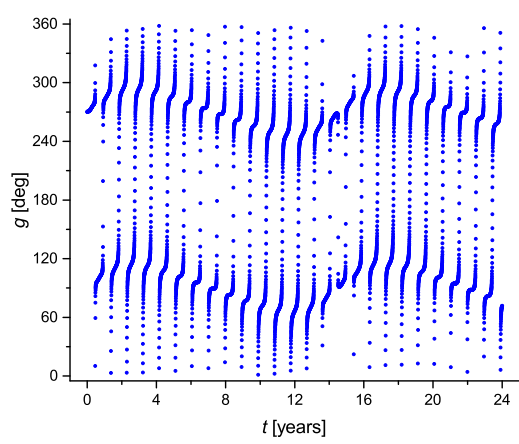

(b)

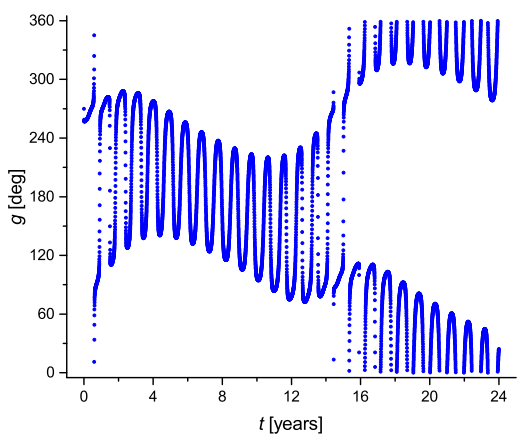

(c)

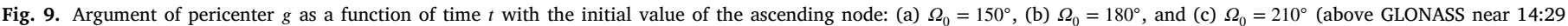
resonance $a_{0}=25947 \mathrm{~km}, i_{0}=64.8^{\circ}, \gamma=30 \mathrm{~m}^{2} / \mathrm{kg}\left(k \gamma=43.2 \mathrm{~m}^{2} / \mathrm{kg}\right)$ ).

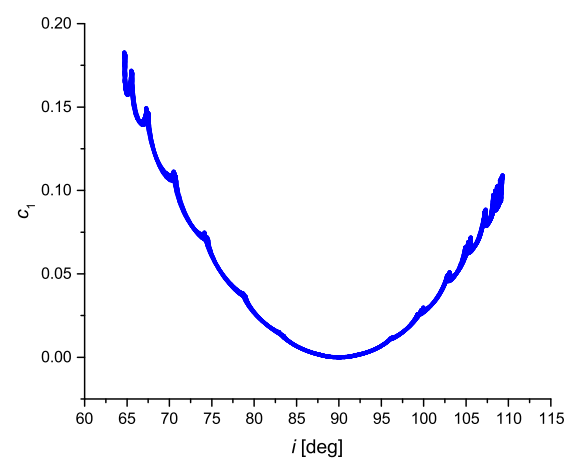

(a)

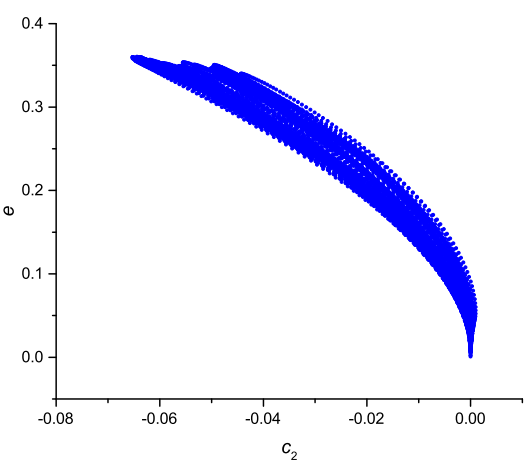

(b)

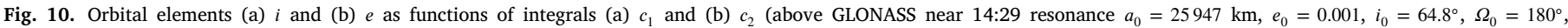
$\left.\gamma=30 \mathrm{~m}^{2} / \mathrm{kg}\left(k \gamma=43.2 \mathrm{~m}^{2} / \mathrm{kg}\right)\right)$.

Further, we have Figs. 9(a) and 9(c), which show us the libration of the argument of pericenter $g$ near $0^{\circ}$ or $360^{\circ}$ passing into libration near the $180^{\circ}$ and vice versa in case of initial values of the longitude of the ascending node $150^{\circ}$ and $210^{\circ}$ correspondingly approximately at the same time when the orbital inclination comes from prograde to retrograde motion. Simultaneously, when the initial value of the longitude of the ascending node is $180^{\circ}$ (Fig. 9(b)) we can see the libration with respect to both points $90^{\circ}$ and $270^{\circ}$.

As shown in Fig. 10, the evolution of the integrals $c_{1}$ and $c_{2}$ (8) is determined by the inclination (Fig. 10(a)) and the eccentricity (Fig. 10(b)), respectively. Moreover, each element is determining for the corresponding integral. In the case of the Lidov-Kozai effect, both elements and the inclination and eccentricity have a comparable effect on the evolution of the integrals. We can conclude that in this case the
Lidov-Kozai effect does not work, since it is suppressed by the action of solar radiation pressure in the case of high area-to-mass ratio objects.

\subsection{Variation of elements and parameters}

Everything that was given earlier was done with fixed initial semimajor axis, initial orbital inclination, and area-to-mass ratio. Now we want to find out how the inclination will evolve depending on the initial inclination, which in turn depends on the satellite navigation system we consider. As a result of modeling, we obtained that with an increase in the initial inclination of the orbit, its maximum inclination will also increase with the growth of the major semi-axis (see Fig. 11).

If we continue the simulation up to the values of the semi-major axes corresponding to the geostationary orbit, then we will get the following. Fig. 12 shows us that the tendency of increase of the maximum orbital 


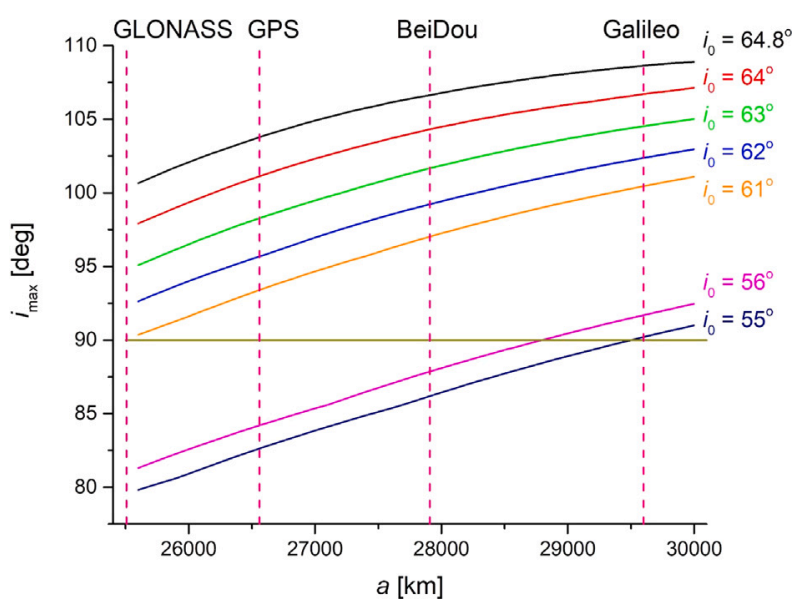

Fig. 11. Dependence of the maximal inclination $i_{\max }$ from the semi-major axis a for different initial values of the inclination with a fixed value of the area-to-mass ratio $\gamma=20 \mathrm{~m}^{2} / \mathrm{kg}\left(\mathrm{k \gamma}=28.8 \mathrm{~m}^{2} / \mathrm{kg}\right)$ and the initial value of the longitude of the ascending node $\Omega=180^{\circ}$.

inclination with increasing of the semi-major axis follows up to the geostationary orbit. Also from Fig. 12, we can emphasize that the amplitude of the flip increases with increasing in the area-to-mass ratio value, because the acceleration induced by the solar radiation pressure increases linearly with respect to the area-to-mass ratio of the space debris. As an illustration, the order of magnitude of the main perturbations as a function of the geocentric distance is represented in [5, Fig. 1]. Near the particular value of $\gamma=15 \mathrm{~m}^{2} / \mathrm{kg}$, the solar radiation pressure equals the acceleration from the Earth's oblateness for an object located at a GNSS altitude. Finally, the solar radiation pressure becomes the major perturbation for objects with sufficiently high area-to-mass ratio, such as $20-30 \mathrm{~m}^{2} / \mathrm{kg}$, after the central body attraction. Which in turn makes it difficult to track such objects in order to prevent their collisions with working satellites.

It was previously mentioned that March 23, 1958, was chosen as the initial epoch. Simulations were also conducted at different dates. Fig. 13 shows the dependency of the maximum inclination of the orbit on the longitude of the ascending node, depending on the area-tomass ratio value at different starting dates with defined semi-major axis $\left(a_{\circ}=25508 \mathrm{~km}\right)$ and initial inclination $\left(i_{\circ}=64.8^{\circ}\right)$. All graphs show that the maximum of inclination is observed at a value of the longitude of the ascending node of $180^{\circ}$.

The tendency to increase inclination with increasing of area-tomass ratio is also noticeable in Fig. 13. This indicates that. that the inclination depends not only on the parameters of the orbit, but also on the parameters of the object itself. Fig. 13(b) gives us two peaks with an area-to-mass ratio of $30 \mathrm{~m}^{2} / \mathrm{kg}$. The appearance of two maxima near $160^{\circ}$ and $210^{\circ}$ is a manifestation of periodic perturbations of the orbital inclination, which are absent at a longitude of $180^{\circ}$. The orange line of Fig. 13(c) contains a gap in itself for the reason that with longitudes of the ascending node from $160^{\circ}$ to $210^{\circ}$ and such a high area-to-mass ratio $\left(\gamma=35 \mathrm{~m}^{2} / \mathrm{kg}\right)$, objects fall to the Earth. Conceptually, these graphs are no different, and this tells us that the initial epoch does not need to be included in the list of parameters for variation. Since a change in the initial epoch simply means a change in the initial position of the bodies relative to each other in space, which, when integrated over an interval 24 or 240 years, does not somehow radically change the results and does not introduce any new laws or dependence on some parameters of the orbit on others.

Table 3 gives the estimations of the minimum area-to-mass ratios $\gamma$ leading to orbital flips for the aforementioned orbits (Table 2). The maximum inclination of the orbits is achieved at initial values of the longitude of the ascending node close to $\Omega=180^{\circ}$ (more generally,
Table 3

Minimum area-to-mass ratios leading to orbital flips at the initial longitude of the ascending node $\Omega_{0}=180^{\circ}$.

\begin{tabular}{lllll}
\hline Orbit & $a[\mathrm{~km}]$ & $i_{0}[\mathrm{deg}]$ & $\gamma\left[\mathrm{m}^{2} / \mathrm{kg}\right]$ & $k \gamma\left[\mathrm{m}^{2} / \mathrm{kg}\right]$ \\
\hline GLONASS & 25508 & 64.8 & 16 & 23.04 \\
Above GLONASS & 25947 & 64.8 & 16 & 23.04 \\
GPS & 26559 & 55 & 25 & 36.00 \\
Above GPS & 27168 & 55 & 24 & 34.56 \\
BeiDou & 27907 & 55 & 22 & 31.68 \\
Above BeiDou & 29034 & 55 & 20 & 28.80 \\
Galileo & 29600 & 56 & 18 & 25.92 \\
Above Galileo & 29994 & 56 & 18 & 25.92 \\
\hline
\end{tabular}

$\Omega+g=\lambda_{S}$ ). We can see that an increase in the area-to-mass ratio $\gamma$ leads to an extension of the range of the longitude of ascending node at which flips occur (see Fig. 13).

The estimations of the minimum area-to-mass ratios $\gamma$ lead to flips of GPS orbits correspond to results [3,4]. The initial conditions C4 and D1 in $[3,4]$ are the most close to $\Omega+g=\lambda_{S}$. The minimum area-to-mass ratio estimation for GPS $k \gamma=36 \mathrm{~m}^{2} / \mathrm{kg}$ agrees with [4, Fig. 20].

The secondary apsidal-nodal secular resonance leads to a couple of evolution between the eccentricity $e$ and the resonant argument $\psi_{1}$ (see Fig. 5). At the initial values of the longitude of the ascending node $\Omega_{0}$ close to $180^{\circ}$, the variation of the initial values of eccentricity up to $e_{0}=0.01$, as well as the initial values of the argument of the pericenter $g_{0}$, does not significantly affect the maximum orbital inclination values. These effects will be considered in more detail in our next work.

\section{Conclusions}

In conclusion, we would like to note that GNSS orbits and possible disposal orbits were examined from the point of view of searching for orbits with minimal variations. There is a dependence of the longperiod evolution of objects with a high area-to-mass ratio on the initial value of the ascending longitude. The maximum inclination of the orbit is achieved when the longitude of the pericenter is sun-synchronous. Flips are possible only for objects with a high area-to-mass ratio $(\gamma>$ $15 \mathrm{~m}^{2} / \mathrm{kg}$ ). Moreover, the flips studied in this work are caused precisely by solar radiation pressure, and not by the Lidov-Kozai effect. The Lidov-Kozai effect is suppressed by solar radiation pressure perturbations, affecting high area-to-mass ratio objects due to a secondary apsidal-nodal secular resonance.

The studied orbits cannot be used as disposal orbits for objects with high area-to-mass ratio or for objects that can produce fragments of space debris with high area-to-mass ratio during storage in disposal orbit.

\section{Declaration of competing interest}

The authors declare that they have no known competing financial interests or personal relationships that could have appeared to influence the work reported in this paper.

\section{Acknowledgments}

We are grateful to anonymous reviewers whose constructive and valuable comments greatly helped us to improve the paper.

This work was supported by the Ministry of Science and Education of the Russian Federation, FEUZ-2020-0030. 


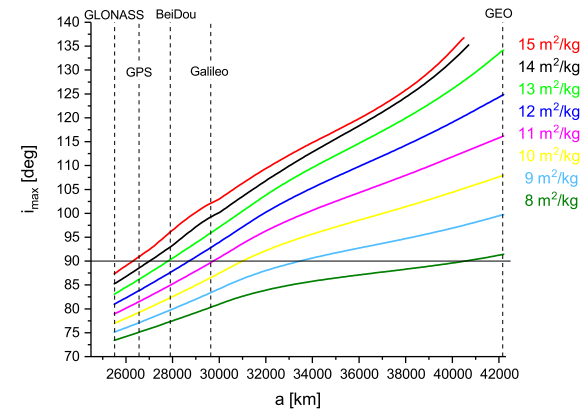

(a)

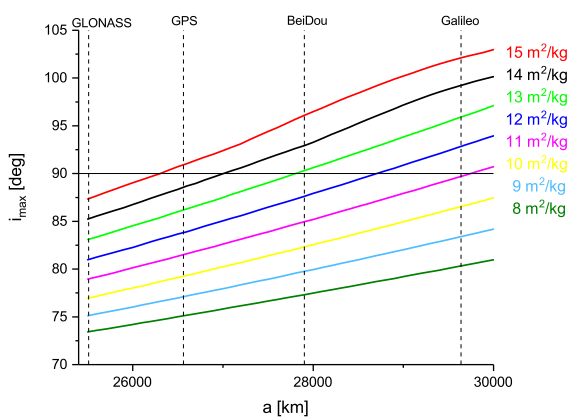

(b)

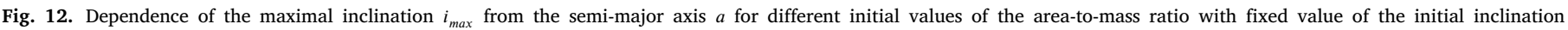
$i_{\circ}=64.8^{\circ}$ and the initial value of the longitude of the ascending node $\Omega_{\circ}=180^{\circ}$.

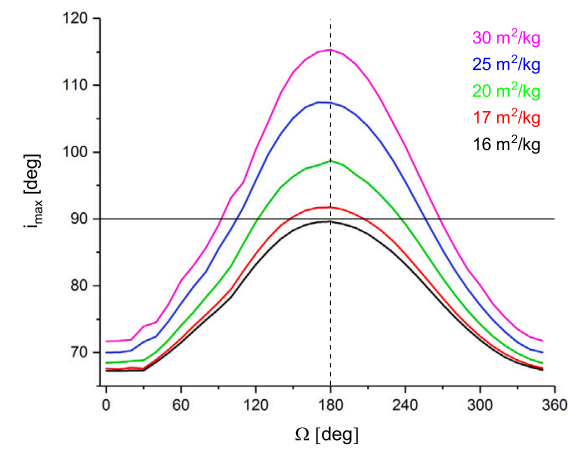

(a)

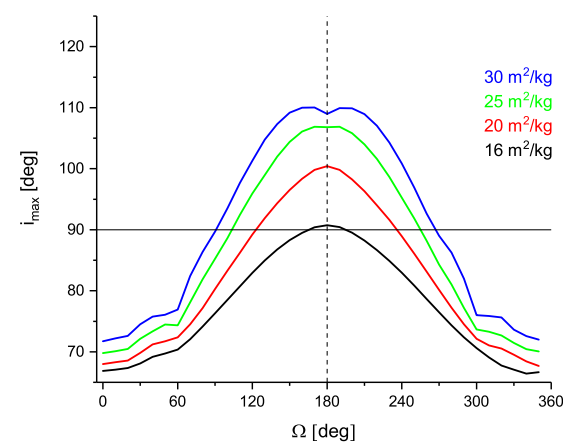

(b)

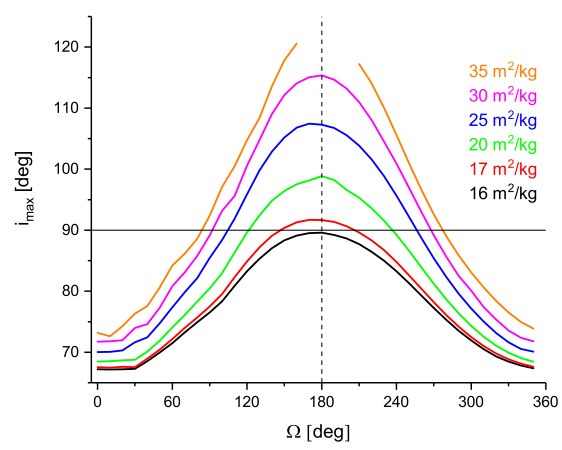

(c)

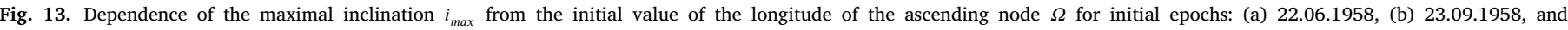

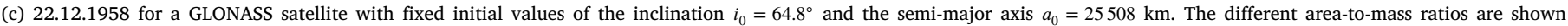
in the legend of the graphs and indicated by color.

\section{References}

[1] I.I. Shevchenko, The Lidov-Kozai effect - Applications in exoplanet research and dynamical astronomy, Astrophys. Space Sci. Libr. 441 (2017) 194

[2] E.D. Kuznetsov, E.A. Avvakumova, Dynamical evolution of space debris in the vicinity of GNSS regions, Acta Astronaut. 158 (2019) 140-147, http://dx.doi. org/10.1016/j.actaastro.2018.02.001.

[3] L. Anselmo, C. Pardini, Dynamical evolution of high area-to-mass ratio debris released into GPS orbits, Adv. Space Res. 43 (10) (2009) 1491-1508, http: //dx.doi.org/10.1016/j.asr.2009.01.017.

[4] L. Anselmo, C. Pardini, Long-term dynamical evolution of high area-to-mass ratio debris released into high earth orbits, Acta Astronaut. 67 (1) (2010) 204-216, http://dx.doi.org/10.1016/j.actaastro.2009.10.017.
[5] S. Valk, A. Lemaître, L. Anselmo, Analytical and semi-analytical investigations of geosynchronous space debris with high area-to-mass ratios, Adv. Space Res. 41 (7) (2008) 1077-1090, http://dx.doi.org/10.1016/j.asr.2007.10.025.

[6] E.D. Kuznetsov, The effect of the radiation pressure on the orbital evolution of geosynchronous objects, Solar Syst. Res. 45 (5) (2011) 433-446, http://dx.doi. org/10.1134/S0038094611050078.

[7] E.D. Kuznetsov, P.E. Zakharova, D.V. Glamazda, A.I. Shagabutdinov, S.O. Kudryavtsev, Light pressure effect on the orbital evolution of objects moving in the neighborhood of low-order resonances, Solar Syst. Res. 46 (6) (2012) 442-449, http://dx.doi.org/10.1134/S0038094612050073.

[8] A.J. Rosengren, D.J. Scheeres, Long-term dynamics of high area-to-mass ratio objects in high-earth orbit, Adv. Space Res. 52 (8) (2013) 1545-1560, http: //dx.doi.org/10.1016/j.asr.2013.07.033. 
[9] D. Casanova, A. Petit, A. Lemaître, Long-term evolution of space debris under the J_2 effect, the solar radiation pressure and the solar and lunar perturbations, Celestial Mech. Dynam. Astronom. 123 (2) (2015) 223-238, http://dx.doi.org/ 10.1007/s10569-015-9644-1.

[10] E.D. Kuznetsov, L.L. Sokolov, Nonlinear evolution of a balloon satellite orbit, Cosm. Res, 39 (6) (2001) 607-614.

[11] L.L. Sokolov, E.D. Kuznetsov, Nonlinear evolution of the orbit eccentricity of a spherically symmetric balloon satellite, Cosm. Res. 44 (6) (2006) 540-547, http://dx.doi.org/10.1134/S0010952506060104.

[12] T.V. Bordovitsyna, I.V. Tomilova, I.N. Chuvashov, The effect of secular resonances on the long-term orbital evolution of uncontrollable objects on satellite radio navigation systems in the MEO region, Solar Syst. Res. 46 (5) (2012) 329-340, http://dx.doi.org/10.1134/S0038094612040016.

[13] C. Lücking, C. Colombo, C.R. McInnes, A passive satellite deorbiting strategy for medium earth orbit using solar radiation pressure and the $\mathrm{J}_{2}$ effect, Acta Astronaut. 77 (2012) 197-206, http://dx.doi.org/10.1016/j.actaastro.2012.03. 026.

[14] A.G. Aleksandrova, T.V. Bordovitsyna, I.V. Tomilova, Investigation of light pressure influence on dynamics of near-earth objects in resonant orbits, Solar Syst. Res. 52 (5) (2018) 435-449, http://dx.doi.org/10.1134/S0038094618050015.

[15] E.M. Alessi, C. Colombo, A. Rossi, Phase space description of the dynamics due to the coupled effect of the planetary oblateness and the solar radiation pressure perturbations, Celestial Mech. Dynam. Astronom. 131 (9) (2019) 43, http://dx.doi.org/10.1007/s10569-019-9919-z, arXiv:1903.09640.

[16] A.V. Krivov, L.L. Sokolov, V.V. Dikarev, Dynamics of mars-orbitting dust: Effects of light pressure and planetary oblateness, Celestial Mech. Dynam. Astronom. 63 (3-4) (1996) 313-339, http://dx.doi.org/10.1007/BF00692293.

[17] E.M. Alessi, G. Schettino, A. Rossi, G.B. Valsecchi, Solar radiation pressure resonances in low earth orbits, Mon. Not. R. Astron. Soc. 473 (2) (2018) 2407-2414, http://dx.doi.org/10.1093/mnras/stx2507, arXiv:1709.09895.
[18] A. Lemaitre, J. Henrard, On the origin of chaotic behavior in the 2/1 Kirkwood gap, Icarus 83 (2) (1990) 391-409, http://dx.doi.org/10.1016/0019-1035(90) 90075-K.

[19] A. Morbidelli, Modern Celestial Mechanics: Aspects of Solar System Dynamics, Taylor and Frances, 2002.

[20] S. Valk, N. Delsate, A. Lemaître, T. Carletti, Global dynamics of high area-tomass ratios GEO space debris by means of the MEGNO indicator, Adv. Space Res. 43 (10) (2009) 1509-1526, http://dx.doi.org/10.1016/j.asr.2009.02.014, arXiv:0810.1859.

[21] A.G. Aleksandrova, T.V. Bordovitsyna, I.N. Chuvashov, Numerical modeling in problems of near-earth object dynamics, Russ. Phys. J. 60 (1) (2017) 80-89, http://dx.doi.org/10.1007/s11182-017-1045-3.

[22] F.G. Lemoine, S.C. Kenyon, J.K. Factor, R.G. Trimmer, N.K. Pavlis, D.S. Chinn, C.M. Cox, S.M. Klosko, S.B. Luthcke, M.H. Torrence, Y.M. Wang, R.G. Williamson, E.C. Pavlis, R.H. Rapp, T.R. Olson, The development of the joint NASA GSFC and National Imagery and Mapping Agency (NIMA) geopotential model EGM96, 1998, NASA/TP-1998-206861.

[23] E. Everhart, Implicit single-sequence methods for integrating orbits, Celest. Mech. 10 (1) (1974) 35-55, http://dx.doi.org/10.1007/BF01261877.

[24] E.M. Standish, JPL Planetary and Lunar Ephemerides, DE405/LE405, Jet Propulsion Laboratory, 1998, Interoffice Memorandum. IOM 312.F-98-048.

[25] C.C. Chao, R.A. Gick, Long-term evolution of navigation satellite orbits: GPS/GLONASS/GALILEO, Adv. Space Res. 34 (5) (2004) 1221-1226, http://dx. doi.org/10.1016/j.asr.2003.01.021.

[26] M.L. Lidov, The evolution of orbits of artificial satellites of planets under the action of gravitational perturbations of external bodies, Planet. Space Sci. 9 (1962) 719-759, http://dx.doi.org/10.1016/0032-0633(62)90129-0.

[27] Y. Kozai, Secular perturbations of asteroids with high inclination and eccentricity, Astron. J. 67 (1962) 579, http://dx.doi.org/10.1086/108876. 